Georgian Mathematical Journal

Volume 12 (2005), Number 2, 321-330

\title{
ARCWISE CONNECTED CONTINUA AND WHITNEY MAPS
}

\author{
IVAN LONČAR
}

\begin{abstract}
Let $X$ be a non-metric continuum, and $C(X)$ be the hyperspace of subcontinua of $X$. It is known that there is no Whitney map on the hyperspace $2^{X}$ for non-metric Hausdorff compact spaces $X$. On the other hand, there exist non-metric continua which admit and ones which do not admit a Whitney map for $C(X)$. In particular, a locally connected or a rimmetrizable continuum $X$ admits a Whitney map for $C(X)$ if and only if it is metrizable. In this paper we investigate the properties of continua $X$ which admit a Whitney map for $C(X)$ or for $C^{2}(X)$.
\end{abstract}

2000 Mathematics Subject Classification: Primary 54F15, 54B20; Secondary 54B35.

Key words and phrases: Arcwise connected continuum, hyperspace, inverse system, property of Kelley, smootness, Whitney map.

\section{INTRODUCTION}

Introduction contains some basic definitions, results and notation.

All spaces in this paper are compact Hausdorff and all mappings are continuous. The weight of a space $X$ is denoted by $w(X)$.

A generalized arc is a Hausdorff continuum with exactly two non-separating points. Each separable arc is homeomorphic to the closed interval $I=[0,1]$.

We say that a space $X$ is arcwise connected provided that for every two distinct points $x, y \in X$ there exists a generalized arc $x y$ with end points $x$ and $y$.

For a compact space $X$ we denote by $2^{X}$ the hyperspace of all nonempty closed subsets of $X$ equipped with the Vietoris topology. $C(X)$ and $X(n)$, where $n$ is a positive integer, stand for the sets of all connected members of $2^{X}$ and of all nonempty subsets consisting of at most $n$ points, respectively, both considered as subspaces of $2^{X}$. The hyperspace $C(C(X))$ is denoted by $C^{2}(X)$.

For a mapping $f: X \rightarrow Y$ define $2^{f}: 2^{X} \rightarrow 2^{Y}$ by $2^{f}(K)=f(K)$ for $K \in$ $2^{X}$. By $[17,5.10] 2^{f}$ is continuous, $2^{f}(C(X)) \subset C(Y)$ and $2^{f}(X(n)) \subset Y(n)$. The restriction $2^{f} \mid C(X)$ is denoted by $C(f)$.

We will use the notion of inverse system as in [7, pp. 135-142]. An inverse system is denoted by $\mathbf{X}=\left\{X_{a}, p_{a b}, A\right\}$. If $\mathbf{X}=\left\{X_{a}, p_{a b}, A\right\}$ is an inverse system, then an element $\left\{x_{a}\right\}$ of the Cartesian product $\prod\left\{X_{a}: a \in A\right\}$ is called a thread of $\mathbf{X}$ if $p_{a b}\left(x_{b}\right)=x_{a}$ for any $a, b \in A$ satisfying $a \leq b$. The subspace of $\prod\left\{X_{a}: a \in A\right\}$ consisting of all threads of $\mathbf{X}$ is called the limit of an inverse system $\mathbf{X}=\left\{X_{a}, p_{a b}, A\right\}$ and is denoted by $\lim \mathbf{X}$ or by $\lim \left\{X_{a}, p_{a b}, A\right\}[7$, p. 135].

ISSN 1072-947X / \$8.00 / (C) Heldermann Verlag www.heldermann.de 
Let $\mathbf{X}=\left\{X_{a}, p_{a b}, A\right\}$ be an inverse system of compact spaces with the natural projections $p_{a}: \lim \mathbf{X} \rightarrow X_{a}$ for $a \in A$. Then $2^{\mathbf{X}}=\left\{2^{X_{a}}, 2^{p_{a b}}, A\right\}, C(\mathbf{X})=$ $\left\{C\left(X_{a}\right), C\left(p_{a b}\right), A\right\}, C^{2}(\mathbf{X})=\left\{C^{2}\left(X_{a}\right), C^{2}\left(p_{a b}\right), A\right\}$ and $\mathbf{X}(n)=\left\{X_{a}(n), 2^{p_{a b}}\right.$ $\left.X_{b}(n), A\right\}$ form inverse systems.

Lemma 1.1 ([10, Lemma 2]). Let $X=\lim \mathbf{X}$. Then $2^{X}=\lim 2^{\mathbf{X}}, C(X)=$ $\lim C(\mathbf{X}), C^{2}(X)=\lim C^{2}(\mathbf{X})$ and $X(n)=\lim \mathbf{X}(n)$.

We say that an inverse system $\mathbf{X}=\left\{X_{a}, p_{a b}, A\right\}$ is $\sigma$-directed if for each sequence $a_{1}, a_{2}, \ldots, a_{k}, \ldots$ of the members of $A$ there is $a \in A$ such that $a \geq a_{k}$ for each $k \in \mathbb{N}$.

In the next we will use the following expanding theorem of non-metric compact spaces into a $\sigma$-directed inverse system of compact metric spaces.

Theorem 1.2. Let $X$ be a compact Hausdorff space such that $w(X) \geq \aleph_{1}$. Then there exists a $\sigma$-directed inverse system $\mathbf{X}=\left\{X_{a}, p_{a b}, A\right\}$ of compact metric spaces $X_{a}$ and surjective bonding mappings $p_{a b}$ such that $X$ is homeomorphic to $\lim \mathrm{X}$. Moreover, if $X$ is a Hausdorff continuum, then each coordinate space $X_{a}$ can be chosen as a metric continuum.

Proof. In [13, Theorem 1.8] it is proved that for a compact Hausdorff space with $w(X) \geq \aleph_{1}$ there exists a $\sigma$-directed inverse system $\mathbf{X}=\left\{X_{a}, p_{a b}, A\right\}$ of compact metric spaces $X_{a}$ such that $X$ is homeomorphic to $\lim \mathbf{X}$. From the proof of $\left[13\right.$, p. 397, Theorem 1.8] it follows that the bonding mappings $p_{a b}$ are surjective. Now, if $X$ is a Hausdorff continuum, then it is clear that each $X_{a}=p_{a}(X)$ is a metric continuum.

The following result [23, p. 173, Problem 23C] will be used.

Theorem 1.3. The following are all equivalent, for a locally compact metric space $X$ :

a) $X$ is separable,

b) $X=\bigcup_{n=1}^{\infty} K_{n}$, where $K_{n}$ is compact and $K_{n} \subset$ Int $K_{n+1}$ for each $n \in \mathbb{N}$,

c) The one point compactification $X^{*}[23$, p. 136] of $X$ is metrizable.

A function $F: X \rightarrow 2^{Y}$ is upper semi-continuous at a point $p \in X$ provided that for every open set $V \subset Y$ such that $F(p) \subset V$ there is an open set $U \subset X$ such that $p \in U$ and satisfying $F(x) \subset V$ for all $x \in U$. The function $F$ is said to be upper semi-continuous if it is upper semi-continuous at each of its points.

We say that a function $F: X \rightarrow 2^{Y}$ is lower semi-continuous at a point $p \in X$ provided that for every open set $G \subset Y$ such that $F(p) \cap G \neq \varnothing$ there exists an open set $U \subset X$ such that $p \in U$ and $F(x) \cap G \neq \varnothing$ for every $x \in U$. The function $F$ is said to be lower semi-continuous if it is lower semi-continuous at each of its points.

\section{A Whitney Map and Hereditarily Irreducible Mappings}

The notion of an irreducible mapping was introduced by Whyburn [22, p. 162]. If $X$ is a continuum, a surjection $f: X \rightarrow Y$ is irreducible provided that no proper subcontinuum of $X$ maps onto all of $Y$ under $f$. 
A mapping $f: X \rightarrow Y$ is said to be hereditarily irreducible [18, p. 204, (1.212.3)] provided that for any given subcontinuum $Z$ of $X$, no proper subcontinuum of $Z$ maps onto $f(Z)$.

A mapping $f: X \rightarrow Y$ is light (zero-dimensional) if all fibers $f^{-1}(y)$ are hereditarily disconnected (zero-dimensional or empty) [7, p. 450], i.e., if $f^{-1}(y)$ does not contain any connected subset of cardinality larger that one ( $\operatorname{dim} f^{-1}(y) \leq 0$ ). Every zero-dimensional mapping is light, and in the realm of mappings with compact fibers the two classes of mappings coincide.

Every hereditarily irreducible mapping is light. If $f: X \rightarrow Y$ is monotone and hereditarily irreducible, then $f$ is one-to-one.

Let $\Lambda$ be a subspace of $2^{X}$. By a Whitney map for $\Lambda$ [18, p. 24, (0.50)] we will mean any mapping $g: \Lambda \rightarrow[0,+\infty)$ satisfying

a) if $A, B \in \Lambda$ such that $A \subset B$ and $A \neq B$, then $g(A)<g(B)$, and

b) $g(\{x\})=0$ for each $x \in X$ such that $\{x\} \in \Lambda$.

If $X$ is a metric continuum, then there exists a Whitney map for $2^{X}$ and $C(X)$ [18, pp. 24-26], [9, p. 106]. If $X$ is a metric continuum, then so is $C(X)$. Hence, there exists a Whitney map for $C^{2}(X)=C(C(X))$. On the other hand, if $X$ is non-metric, then it admits no Whitney map for $2^{X}[2, \mathrm{p}$. 305]. It is known that there exist non-metric continua which admit and ones which do not admit a Whitney map for $C(X)$ [2, p. 307]. Moreover, if $X$ is a non-metric locally connected or a rim-metrizable continuum, then $X$ admits no Whitney map for $C(X)$ [12, Theorem 8 and 11].

The following external characterization of non-metric continua which admit a Whitney map is proved in [13, p. 399, Theorem 2.3] for continua, but the proof given in [13, p. 399, Theorem 2.3] can be applied without essential changes to compact spaces.

Theorem 2.1. Let $X$ be a compact space. Then $X$ admits a Whitney map for $C(X)$ if and only if for each $\sigma$-directed inverse system $\mathbf{X}=\left\{X_{a}, p_{a b}, A\right\}$ of compact spaces $X_{a}$ which admit Whitney maps for $C\left(X_{a}\right)$ and $X=\lim \mathbf{X}$, there exists a subset $B$ of $A$ cofinal in $A$ and such that, for each $b \in B$, the projection $p_{b}: X \rightarrow X_{b}$ is hereditarily irreducible. Moreover, for the necessity the condition that each space $X_{a}$ admits a Whitney map for $C\left(X_{a}\right)$ is not required.

We say that a continuum $X$ admits a Whitney map for $C^{2}(X)$ if there is a mapping $h: C^{2}(X) \rightarrow[0,+\infty)$ such that

c) if $\mathcal{A}, \mathcal{B} \in C^{2}(X)$ are such that $\mathcal{A} \subset \mathcal{B}$ and $\mathcal{A} \neq \mathcal{B}$, then $h(\mathcal{A})<h(\mathcal{B})$ and

d) $h(\mathcal{D})=0$ for each $\mathcal{D} \in(X(1))(1)$.

Theorem 2.2. If a continuum $X$ admits a Whitney map for $C^{2}(X)$, then $X$ admits a Whitney map for $C(X)$.

Proof. Since $X$ admits a Whitney map for $C^{2}(X)$ there is a mapping $h$ : $C^{2}(X) \rightarrow[0,+\infty)$ such that: c) if $\mathcal{A}, \mathcal{B} \in C^{2}(X)$ are such that $\mathcal{A} \subset \mathcal{B}$ and $\mathcal{A} \neq \mathcal{B}$, then $h(\mathcal{A})<h(\mathcal{B})$ and d) $h(\{\{x\}\})=0$ for each $x \in X$. Given $A \in C(X)$ it follows that $A(1) \in C^{2}(X)$. Thus we can consider the function 
$\mu: C(X) \rightarrow[0,+\infty)$ defined by $\mu(A)=h(A(1))$, for any $A \in C(X)$. Let $A, B \in C(X)$ be such that $A \subset B$ and $A \neq B$. Then $A(1), B(1) \in C^{2}(X)$ are such that $A(1) \subset B(1)$ and $A(1) \neq B(1)$, By c) we have $h(A(1))<h(B(1))$. This implies that $\mu(A)<\mu(B)$. Now, let $x \in X$. By d) $\mu(\{x\})=h(\{x\}(1))=$ $h(\{\{x\}\})=0$. Using the Vietoris topology on both $C(X)$ and $C^{2}(X)$ it can be shown that $\mu$ is continuous. Thus, $X$ admits a Whitney map for $C(X)$.

We say that a continuum $C(X)$ admits a Whitney map for $C^{2}(X)$ if there is a mapping $f: C^{2}(X) \rightarrow[0,+\infty)$ such that

e) if $\mathcal{A}, \mathcal{B} \in C^{2}(X)$ are such that $\mathcal{A} \subset \mathcal{B}$ and $\mathcal{A} \neq \mathcal{B}$, then $f(\mathcal{A})<f(\mathcal{B})$ and

d) $f(\mathcal{D})=0$ for each $\mathcal{D} \in C(X)(1)$.

Theorem 2.3. Let $X$ be a continuum. If $C(X)$ admits a Whitney map for $C^{2}(X)$, then $X$ admits a Whitney for $C(X)$.

Proof. Let $\mathbf{X}=\left\{X_{a}, p_{a b}, A\right\}$ be a $\sigma$-directed inverse system such that each coordinate space $X_{a}$ is a continuum which admits a Whitney map for $C\left(X_{a}\right)$ and $X=\lim \mathbf{X}$. Then $C(\mathbf{X})=\left\{C\left(X_{a}\right), C\left(p_{a b}\right), A\right\}$ is a $\sigma$-directed inverse system such that $C(X)$ is homeomorphic to $\lim C(\mathbf{X})$ (Lemma 1.1). Since $C(X)$ admits a Whitney map for $C^{2}(X)$, we can apply the necessity of Theorem 2.1 to $C(X)$ in place of $X$ to conclude that there is a subset $B$ of $A$ cofinal in $A$ such that the projections $C\left(p_{b}\right): C(X) \rightarrow C\left(X_{b}\right)$ are hereditarily irreducible. Now, the restriction $C\left(p_{b}\right) \mid X(1)$ is again hereditarily irreducible since $X(1)$ is a subcontinuum of $C(X)$. Let us observe that $\left(C\left(p_{b}\right) \mid X(1)\right)(X(1)) \subset X_{b}(1)$ and that both $X(1)$ and $X_{b}(1)$ are homeomorphic to $X$ and $X_{b}$, respectively. Thus $C\left(p_{b}\right) \mid X(1)=p_{b}$, which means that the projections $p_{b}: X \rightarrow X_{b}$ are hereditarily irreducible, for each $b \in B$. Finally, from Theorem 2.1 it follows that $X$ admits a Whitney map for $C(X)$.

\section{The Metrizability of $C(X) \backslash X(1)$ If $X$ is Arcwise Connected}

Now we will prove the metrizability of $C(X) \backslash X(1)$ if $X$ is an arcwise connected continuum which admits a Whitney map for $C(X)$.

Theorem 3.1. If an arcwise connected continuum $X$ admits a Whitney map for $C(X)$, then $C(X) \backslash X(1)$ is metrizable and $w(C(X) \backslash X(1)) \leq \aleph_{0}$.

Proof. Assume that a non-metric arcwise connected continuum $X$ admits a Whitney map for $C(X)$. From Theorem 1.2 it follows that there exists a $\sigma$ directed inverse system $\mathbf{X}=\left\{X_{a}, p_{a b}, A\right\}$ of metric continua and surjective bonding mappings such that $X$ is homeomorphic to $\lim \mathbf{X}$. Consider the inverse system $C(\mathbf{X})=\left\{C\left(X_{a}\right), C\left(p_{a b}\right), A\right\}$ whose limit is $C(X)$ (Lemma 1.1). From Theorem 2.1 it follows that there exists a subset $B$ cofinal in $A$ such that the projections $p_{b}$ are hereditarily irreducible. By $[18$, p. 204, (1.212.3)] the hereditarily irreducibility of $p_{b}$ implies that $C\left(p_{b}\right)$ is light for every $b \in B$. Since $\lim \mathbf{X}$ is homeomorphic to $\lim \left\{X_{b}, p_{b c}, B\right\}$, we may assume that $B=A$. Let $Y_{a}=C\left(p_{a}\right)(C(X))$. Furthermore, $C\left(p_{a}\right)^{-1}\left(X_{a}(1)\right)=X(1)$ since from the 
hereditary irreducibility of $p_{a}$ it follows that no non-degenerate subcontinuum of $X$ maps under $p_{a}$ onto a point. We infer that $C\left(p_{a}\right)^{-1}\left[Y_{a} \backslash X_{a}(1)\right]=C(X) \backslash X(1)$. Let us prove that the restriction $C\left(p_{a}\right) \mid[C(X) \backslash X(1)]$ is one-to-one. Suppose that $C\left(p_{a}\right) \mid[C(X) \backslash X(1)]$ is not one-to-one. Then there exists a continuum $C_{a}$ in $X_{a}$ and two non-degenerate and distinct continua $C, D$ in $X$ such that $p_{a}(C)=p_{a}(D)=C_{a}$. It is impossible that $C \subset D$ or $D \subset C$ since $p_{a}$ is hereditarily irreducible. Otherwise, if $C \cap D \neq \varnothing$, then for the continuum $Y=C \cup D$ we have that $C$ and $D$ are proper subcontinua of $Y$ and $p_{a}(Y)=$ $p_{a}(C)=p_{a}(D)=C_{a}$, which is impossible since $p_{a}$ is hereditarily irreducible. We infer that $C \cap D=\varnothing$. There exists a generalized arc $E$ with end points in $C$ and $D$, respectively. Moreover, since $C$ and $D$ are non-degenerate, we may assume that $E \cap C \neq C$ and $E \cap D \neq D$. Now $p_{a}(E \cup D)=p_{a}(E)$, which is impossible since $p_{a}$ is hereditarily irreducible. It follows that the restriction $P_{a}=C\left(p_{a}\right) \mid[C(X) \backslash X(1)]$ is one-to-one and closed [7, p. 95, Proposition 2.1.4]. Hence, $P_{a}$ is a homeomorphism and $C(X) \backslash X(1)$ is metrizable. Moreover, $w(C(X) \backslash X(1)) \leq \aleph_{0}$ since $Y_{a}$ as a compact metrizable space is separable and, consequently, second-countable [7, p. 320].

It is known that if $X$ is a continuum, then $C(X)$ is arcwise connected [16, p. 1209, Theorem]. Hence, we have the following corollary.

Corollary 3.2. If $X$ is a continuum which admits a Whitney map for the hyperspace $C^{2}(X)$, then $C^{2}(X) \backslash C(X)(1)$ is metrizable and $w\left(C^{2}(X) \backslash C(X)(1)\right)$ $\leq \aleph_{0}$.

We close this section with the following result.

Theorem 3.3. If an arcwise connected continuum $X$ admits a Whitney map for the hyperspace $C(X)$, then $C(X) \backslash X(1)$ admits a Whitney map for both $C(C(X) \backslash X(1))$ and $(C(X) \backslash X(1))(1)$.

Proof. By Theorem 3.1 the space $C(X) \backslash X(1)$ is metrizable and $w(C(X) \backslash X(1))$ $\leq \aleph_{0}$. This means that $C(X) \backslash X(1)$ is separable. Now we will use the theorem due to T. Watanabe [21, Theorem 1] which states that if $Z$ is a separable metric space, then $Z$ admits a Whitney map $\mu: 2^{Z} \rightarrow \mathbb{R}$. This means that there exists a Whitney map $v$ for $2^{C(X) \backslash X(1)}$. The restrictions of $v$ to $C(C(X) \backslash X(1))$ and $(C(X) \backslash X(1))(1)$ are Whitney maps as well.

\section{Smoothness and Whitney Maps}

There are many definitions of smoothness in the literature. The following concept of smoothness is due to Maćkowiak [15] for metric continua and to Rakowski [19] for Hausdorff continua. We call this concept the MR-smoothness.

4.1. MR-smoothness. We say that a pointed continuum $(X, p)$ is $M R$-smooth provided that $X$ is smoth at $p$, i.e., for each subcontinuum $L$ of $X$ which contains $p$ and for each open set $V$ which contains $L$ there exists an open connected set $U$ such that $L \subset U \subset V$ [3, p. 103]. 
For a given pointed continuum $(X, p)$ consider a function $\delta_{(X, p)}: X \rightarrow C^{2}(X)$ defined by

$$
\delta_{(X, p)}(x)=\{K \in C(X): p, x \in K\} .
$$

The following two theorems have been proved in the metric case [5, Propositions 1 and 2] respectively, but they remain valid for Hausdorff continua [3, p. 103].

Theorem 4.1. The function $\delta_{(X, p)}$ is upper semi-continuous.

Theorem 4.2 ([3, Theorem 8.1]). The function $\delta_{(X, p)}$ is continuous if and only if the pointed continuum $(X, p)$ is MR-smooth.

Lemma 4.3. If $(X, p)$ is a pointed arcwise connected $M R$-smooth continuum, then $\delta_{(X, p)}: X \rightarrow C^{2}(X)$ is an embedding and $\delta_{(X, p)}(X) \subset C^{2}(X) \backslash C(X)(1)$.

Proof. According to Theorem 4.2 the function $\delta_{(X, p)}: X \rightarrow C^{2}(X)$ is continuous since $(X, p)$ is MR-smooth. Moreover, if $x \neq y$, then the generalized arcs $p x$ and $p y$ are distinct, whence $\{K \in C(X): p, x \in K\} \neq\{K \in C(X): p, y \in K\}$. This means then $\delta_{(X, p)}$ is one-to-one. Thus it is an embedding. Let us prove that $\delta_{(X, p)}(X) \subset C^{2}(X) \backslash C(X)(1)$. If $X$ is not a generalized arc, then $\delta_{(X, p)}(x)=$ $\{K \in C(X): p, x \in K\}$ is a non-degenerate continuum in $C(X)$ which contains a generalized arc $p x$ and $X$. Hence, $\delta_{(X, p)}(x) \in C^{2}(X) \backslash C(X)(1)$. If $X$ is a generalized arc, then $X$ is MR-smooth at each of its points. We may assume that $p$ is not an end point. This implies that $\delta_{(X, p)}(x)$ is a non-degenerate continuum in $C(X)$ which contains a generalized arc $p x$ and $X$.

Theorem 4.4. If $(X, p)$ is a pointed arcwise connected $M R$-smooth continuum which admits a Whitney map for $C^{2}(X)$, then $X$ is metrizable.

Proof. From Corollary 3.2 it follows that $C^{2}(X) \backslash C(X)(1)$ is metrizable and $w\left(C^{2}(X) \backslash C(X)(1)\right) \leq \aleph_{0}$ since $C(X)$ is arcwise connected. Using Lemma 4.3 we infer that $\delta_{(X, p)}(X) \subset C^{2}(X) \backslash C(X)(1)$. Hence $X$ is metrizable and separable.

Corollary 4.5. An MR-smooth arcwise connected pointed continuum $(X, p)$ admits a Whitney map for $C^{2}(X)$ if and only if it is metrizable.

An arboroid is a hereditarily unicoherent continuum which is arcwise connected. A metrizable arboroid is a dendroid. If $X$ is an arboroid and $x, y \in X$, then there exists a unique generalized arc $x y$ in $X$ with end points $x$ and $y$.

Corollary 4.6. An MR-smooth pointed arboroid $(X, p)$ admits a Whitney map for $C^{2}(X)$ if and only if it is metrizable.

Proof. Apply Theorem 4.4.

4.2. Arc-smoothness. The notion of arc-smoothness was introduced by Fugate, Gordh and Lum in [8]. We will use the generalization of this notion from $[11]$.

An arc-structure on a continuum $X[11$, p. 172] is a function $A: X \times X \rightarrow$ $C(X)$ such that for $x \neq y$ in $X$, the set $A(x, y)$ is a generalized arc from $x$ to $y$ 
and such that the following metric-like conditions are satisfied for all $x, y$ and $z$ in $X$ :

(a) $A(x, x)=\{x\}$,

(b) $A(x, y)=A(y, x)$, and

(c) $A(x, z) \subseteq A(x, y) \cup A(y, z)$ with equality prevailing whenever $y$ belongs to $A(x, z)$.

The pair $(X, A)$ is arc-smooth at point $p$ in $X$ if the induced function $A_{p}$ : $X \rightarrow C(X)$ defined by $A_{p}(x)=A(p, x)$ is continuous. The pair $(X, A)$ is arc-smooth if there exists a point in $X$ at which $(X, A)$ is arc-smooth.

Remark. In [14] the set $A_{p}(X)$ is denoted by $\mathcal{D}(X, p)$ and it is proved that if $X$ is smooth at $p$, then $\mathcal{D}(X, p)$ is arcwise connected [14, Theorem 4.8]. Moreover, if the continuum $X$ is arcwise connected and smooth at a point $p$, then there exists a homeomorphism $h: X \rightarrow \mathcal{D}(X, p)$ [14, Theorem 8.2]. Namely, $h$ is defined by $h(x)=A(p, x)$.

If a continuum $X$ is arc-smooth at the point $p$, then $A_{p}: X \rightarrow C(X)$ is one-to-one. Thus, we have the following lemma.

Lemma 4.7. Let $X$ be a continuum with an arc-structure $A$. If $(X, A)$ is arc-smooth at a point $p \in X$, then $A_{p}(X) \subset C(X)$ is homeomorphic to $X$.

Now we are ready to prove the following theorem.

Theorem 4.8. If $X$ is an arc-smooth continuum, then $X$ admits a Whitney map for $C(X)$ if and only if $X$ is metrizable.

Proof. It is known that if $X$ is metrizable, then $X$ admits a Whitney map for $C(X)$. Suppose that $X$ is non-metrizable and there exists a Whitney map for $C(X)$. Let $X$ be arc-smooth at a point $p$. By Lemma $4.7 X$ is homeomorphic to $A_{p}(X) \subset C(X)$. It is clear that $A_{p}(X) \backslash\{\{p\}\} \subset C(X) \backslash X(1)$. We infer that $A_{p}(X) \backslash\{\{p\}\}$ is metrizable since $C(X) \backslash X(1)$ is metrizable (Theorem 3.1). Hence $X \backslash\{p\}$ is metrizable since it is homeomorphic to $A_{p}(X) \backslash\{\{p\}\}$ under the homeomorphism $h(x)=A(p, x)$. Moreover, from Theorem 3.1 it follows that $X$ is separable since $w(C(X) \backslash X(1)) \leq \aleph_{0}$ and $A_{p}(X) \backslash\{\{p\}\} \subset C(X) \backslash X(1)$. Furthermore, $X$ is the one point compactification of $X \backslash\{p\}$. Finally, from Theorem 1.3 it follows that $X$ is metrizable, a contradiction.

Formerly the smoothness was defined for fans [1, p. 7] and for metric arboroids, i.e., for dendroids [4, p. 298, Definition].

An arboroid $X$ is said to be smooth if there exists a point $p \in X$, called an initial point of $X$, such that for every convergent net $\left\{a_{d}: d \in D\right\}$ of points $a_{d}$ of $X$ the condition $\lim \left\{a_{d}: d \in D\right\}=a$ implies that the net of $\operatorname{arcs}\left\{p a_{d}: d \in D\right\}$ is convergent and $\operatorname{Lim}\left\{p a_{d}: d \in D\right\}=p a$.

Lemma 4.9 ([8, p. 647]). An arboroid is smooth if and only if it is arcsmooth.

Theorem 4.10. If a smooth arboroid $X$ admits a Whitney map for $C(X)$, then $X$ is metrizable. 


\section{The Property of Kelley}

We say that a continuum $X$ has the property of Kelley at a point $p \in X$ if for every subcontinuum $K \subset X$ containing $p$ and for every open neighborhood $\mathcal{U}$ of $K$ in the hyperspace $C(X)$, there exists a neighborhood $U$ of $p$ in $X$ such that if $q \in U$ then there is a continuum $L \in C(X)$ with $q \in L \in \mathcal{U}$. A continuum $X$ has the property of Kelley if it has the property of Kelley at each of its points.

For a given continuum $X$ we define the function $\alpha_{X}: X \rightarrow C^{2}(X)$ by

$$
\alpha_{X}(x)=\{A \in C(X): x \in A\}
$$

for each point $x \in X[3$, p. 91].

Lemma 5.1. The function $\alpha_{X}$ is upper semi-continuous.

Proof. See [20, p. 292, (2.1) Theorem].

Theorem 5.2 ([3, Theorem 3.1]). The function $\alpha_{X}$ is continuous if and only if $X$ has the property of Kelley.

Hence we have the following lemma.

Lemma 5.3. If a continuum $X$ has the property of Kelley, then the function $\alpha_{X}: X \rightarrow C^{2}(X) \backslash C(X)(1)$ is an embedding.

Proof. Let us note that

$$
\alpha_{X}(X) \subset C^{2}(X) \backslash C(X)(1) .
$$

The rest follows from Theorem 5.2.

Now we are ready to prove the following theorem.

Theorem 5.4. If a continuum $X$ with the property of Kelley admits a Whitney map for $C^{2}(X)$, then it is metrizable.

Proof. By Corollary 3.2 the set $C^{2}(X) \backslash C(X)(1)$ is metrizable and

$$
w\left(C^{2}(X) \backslash C(X)(1)\right) \leq \aleph_{0} .
$$

Using Lemma 5.3 we see that $\alpha_{X}(X) \subset C^{2}(X) \backslash C(X)(1)$ is metrizable. Moreover, $X$ is homeomorphic to $\alpha_{X}(X)$. Hence $X$ is metrizable.

Problem 1. Is it true that a continuum $X$ with the property of Kelley is metrizable if it admits a Whitney map for $C(X)$ ?

We say that a continuum $X$ is hereditarily indecomposable if no subcontinuum of $X$ can be written as the union of two proper subcontinua [9, p. 61].

Lemma 5.5 ([6, p. 211, Proposition 2.7]). Hereditarily indecomposable continua have the property of Kelley.

From Theorem 5.4 we obtain the following result.

Theorem 5.6. If a hereditarily indecomposable continuum $X$ admits a Whitney map for $C^{2}(X)$, then $X$ is metrizable. 


\section{Concluding Remarks}

It is known [7, p. 171, Corollary 3.1.20] that if a compact space $X$ is a countable union of its subspaces $X_{n}, n \in \mathbb{N}$, such that $w\left(X_{n}\right) \leq \aleph_{0}$, then $w(X) \leq \aleph_{0}$. Using this fact and the theorems proved in the previous sections we obtain the following results.

Theorem 6.1. If a continuum $X$ is the countable union of its arcwise connected MR-smooth continua and if $X$ admits a Whitney map for $C^{2}(X)$, then $X$ is metrizable.

Proof. Apply Theorem 4.4.

Theorem 6.2. If a continuum $X$ is the countable union of its arc-smooth continua and if $X$ admits a Whitney map for $C(X)$, then $X$ is metrizable.

Proof. Apply Theorem 4.8.

Finally, applying Theorem 5.4 we obtain the following theorem.

Theorem 6.3. If a continuum $X$ is the countable union of its subcontinua with the property of Kelley and if $X$ admits a Whitney map for $C^{2}(X)$, then $X$ is metrizable.

\section{ACKNOWLEDGEMENT}

The author is very grateful to the referee for his/her valuable suggestions.

\section{REFERENCES}

1. J. J. Charatonik, On fans. Dissertationes Math. (Rozprawy Mat.) 54(1967), 39 pp.

2. J. J. Charatonik and W. J. Charatonik, Whitney maps - a non-metric case. Colloq. Math. 83(2000), No. 2, 305-307.

3. J. J. Charatonik and W. J. Charatonik, Connections between classes of spaces and of mappings. Proceedings of the 2000 Topology and Dynamics Conference (San Antonio, TX). Topology Proc. 25(2000), Spring, 87-111.

4. J. J. Charatonik and C. Eberhart, On smooth dendroids. Fund. Math. 67(1970), $297-322$.

5. W. J. Charatonik, Inverse limits of smooth continua. Comment. Math. Univ. Carolin. 23(1982), No. 1, 183-191.

6. W. J. Charatonik, A homogeneous continuum without the property of Kelley. Topology Appl. 96(1999), No. 3, 209-216.

7. R. Engelking, General topology. Translated from the Polish by the author. Monografie Matematyczne, Tom 60. [Mathematical Monographs, Vol. 60]. PWN-Polish Scientific Publishers, Warsaw, 1977.

8. J. B. Fugate, G. R. Gordh, JR., and Lewis Lum, On arc-smoth continua. Topology Proc. 2(1977), 645-656.

9. A. Illanes and S. B. Nadler, JR., Hyperspaces. Fundamentals and recent advances. Monographs and Textbooks in Pure and Applied Mathematics, 216. Marcel Dekker, Inc., New York, 1999.

10. Y. Kodama, S. Spiez, and T. Watanabe, On shape of hyperspaces. Fund. Math. 100(1978), No. 1, 59-67. 
11. A. Koyama, Weakly arc-smooth continua. I. Glas. Mat. Ser. III 22(42)(1987), No. 1, 171-185.

12. I. LonČAR, A note on a Whitney map for continua. Math. Commun. 6(2001), No. 1, 1-9.

13. I. LonČAR, A fan $X$ admits a Whitney map for $C(X)$ iff it is metrizable. Glas. Mat. Ser. III 38(58)(2003), 395-411.

14. L. Lum, Weakly smooth continua. Trans. Amer. Math. Soc. 214(1975), 153-167.

15. T. MaĆKowiak, On smooth continua. Fund. Math. 85(1974), No. 1, 79-95.

16. M. M. McWaters, Arcs, semigroups, and hyperspaces. Canad. J. Math. 20(1968), 1207-1210.

17. E. Michael, Topologies on spaces of subsets. Trans. Amer. Math. Soc. 71(1951), 152182.

18. S. B. NAdLER, JR., Hyperspaces of sets. A text with research questions. Monographs and Textbooks in Pure and Applied Mathematics, 49. Marcel Dekker, Inc., New York-Basel, 1978.

19. Z. M. Rakowski, Smooth Hausdorf continua. Bull. Acad. Polon. Sci. Sér. Sci. Math. Astronom. Phys. 25(1977), No. 6, 563-566.

20. R. W. Wardle, On a property of J. L. Kelley. Houston J. Math. 3(1977), No. 2, 291-299.

21. T. Watanabe, A Whitney map and the Lindelöf property. Houston J. Math. 15(1989), No. 1, 147-151.

22. G. T. Whyburn, Analytic topology. American Mathematical Society Colloquium Publications, XXVIII. American Mathematical Society, Providence, R.I., 1963.

23. S. Willard, General topology. Addison-Wesley Publishing Co., Reading, Mass.-LondonDon Mills, Ont., 1970.

(Received 4.12.2003; revised 3.02.2005)

Author' address:

Faculty of Organization and Informatics Varaždin

(University of Zagreb, Croatia)

Pavlinska 2, HR-42000 Varaždin

Croatia

E-mails: ivan.loncar1@vz.tel.hr

ivan.loncar@foi.hr 